\title{
The role of unenhanced phase of the liver in the scanning protocol of metastatic breast cancer: implications for sensitivity, response evaluation and size measurement
}

\author{
Juan José Arenas-Jiménez, Elena García-Garrigós, Mariana Cecilia Planells-Alduvín
}

Department of Radiology, Hospital General Universitario de Alicante, Instituto de Investigación Sanitaria y Biomédica de Alicante (ISABIAL), FISABIO Hospital General Universitario de Alicante, Alicante, Spain

Radiol Oncol 2021; 55(4): 418-425.

Received 13 February 2021

Accepted 1 May 2021

Correspondence to: Juan José Arenas-Jiménez, Ph.D., Department of Radiology, Hospital General Universitario de Alicante, Instituto de Investigación Sanitaria y Biomédica de Alicante (ISABIAL), FISABIO Hospital General Universitario de Alicante, Av Pintor Baeza 12, 03010, Alicante, Spain 03560. E-mail: j.arenasjimenez@gmail.com

Disclosure: No potential conflicts of interest were disclosed.

This is an open access article under the CC BY-NC-ND license (http://creativecommons.org/licenses/by-nc-nd/4.0/).

Background. To analyse if performing unenhanced CT of the liver aids in the evaluation of metastatic lesions, response assessment or alter the size of the lesions, compared with portal phase alone, in patients with hepatic metastases from breast carcinoma.

Patients and methods. One-hundred and fifty-three CT scans of 36 women were included. Scans consisted of unenhanced, arterial and portal delayed phases of the liver. Two readers sorted which phase was best for visualization of metastases, evaluated the number of lesions detected in each phase, selected the best phase for assessment of response in two consecutive scans, and measured one target lesion in all the phases. $X^{2}$ was used to compare differences among phases and paired $t$ test for measurement differences.

Results. Unenhanced, arterial and portal phases were considered better phases by readers $1 / 2$ in $68 / 67 \%, 27 / 28 \%$ and $69 / 70 \%$, and some lesions were missed in $2 \%, 11 \%$ and $7 \%$, respectively. Sensitivity was significantly better for unenhanced and portal phases compared to arterial phase. Comparison between consecutive scans was considered better in unenhanced (80/79\%), followed by portal $(70 / 69 \%)$ and arterial phases $(31 / 31 \%)$. Maximum diameter of target lesions was $15 \%$ greater in unenhanced phase $(p<0.001)$.

Conclusions. Portal and unenhanced phases of the liver allow better detection and delineation of metastatic hepatic lesions from breast carcinoma. In most cases, unenhanced CT is the best phase to assess response and provides the largest diameter. Therefore, we recommend the use of unenhanced $\mathrm{CT}$ in the evaluation of patients with breast carcinoma and suspected or known hepatic metastatic disease.

Key words: breast cancer; staging; computed tomography; metastases; hepatic lesion

\section{Introduction}

Breast cancer is the neoplasm with the highest incidence and mortality rates among women worldwide, and hepatic metastases appear in more than $50 \%$ of patients with advanced disease. ${ }^{1,2}$

Conspicuity and detection of lesions in multiple phases of contrast enhanced CT of the liver has been evaluated in patients with metastases. ${ }^{3,6}$ The need of unenhanced CT for detection of metastases has been debated from the early times of oncologic applications of $\mathrm{CT}^{7,8}$, with unenhanced phase being used for scanning breast cancer patients in $21 \%$ of the institutions in a survey. ${ }^{9}$

Some studies evaluate the need of multiple phases of dynamic CT of the liver for a better de- 
tection of hepatic metastases from breast cancer, but they were performed in the 1990s, with much different technological equipment and therapeutic schemes compared to those used nowadays. $3,7,8,10,11$ In those studies, unenhanced CT added little to the enhanced phases in terms of the amount of lesions detected, although it was demonstrated that some lesions are only or better seen in that phase. Moreover, it has been proved that the size of the lesions is different in unenhanced CT compared to other phases, with one study concluding that the unenhanced CT depicts the maximum volume of hepatic metastases. ${ }^{3}$ More recently, a Critical Appraised Topic on this subject ${ }^{12}$ addressed the need of confirmation of the role of unenhanced CT with modern technology and, more importantly, remarked the need to evaluate the confidence of radiologists to delineate the lesions, that plays a major role in measurement and response evaluation.

In this setting, our hypothesis was that, in patients with breast cancer, unenhanced $\mathrm{CT}$, even with modern equipment, could add information to enhanced CT of the liver in patients with hepatic metastases by increasing the detection of lesions, permitting greater confidence for delineation of the metastases and providing a more accurate depiction of the size of them. For this purpose, we conducted this study, in a series of patients with breast cancer and hepatic metastases detected by $\mathrm{CT}$, comparing tumor conspicuity, sensitivity, comparison on follow-up examination and size of hepatic metastasis in unenhanced, arterial and portal phases of the liver.

\section{Patients and methods}

This retrospective observational study was reviewed and approved by the institutional review board and local ethics committee. Informed consent was waived since no intervention was made on the routine institutional protocol for the purpose of the study.

\section{Patient population}

From May 2016 to February 2018, patients with already known or newly diagnosed hepatic metastases from breast cancer referred to our department for a $\mathrm{CT}$, either as initial staging or for follow-up, were consecutively included. Patients were excluded if CT wasn't performed according to the scan protocol detailed below, if contrast injection protocol couldn't be achieved as described, or if they weighted less than 50 or more than 100 $\mathrm{kg}$. A total of 48 patients with hepatic metastases were scanned during that period. Of them, 12 were excluded for the following reasons: contrast protocol couldn't be achieved in 9 patients due to either renal insufficiency or inadequate vein access, 2 for weighing more than $100 \mathrm{~kg}$ and one less than $50 \mathrm{~kg}$. Finally, 153 examinations in 36 women (range1-7) were included, 4 were the initial examination and the remaining 149 were follow-up. Mean age was 59.2 years, range $34-77$. Histological confirmation of metastases was available in 2 patients, in the rest, clinical and radiological diagnosis was established taking into consideration radiological presentation and follow-up. No case had to be excluded due to diagnostic uncertainty for presenting indeterminate lesions posing diagnostic doubts at follow-up.

\section{CT protocols}

All CT scans were obtained with a 64-MDCT scanner (Philips Brilliance 64, Philips Medical Systems, Cleveland, $\mathrm{OH}, \mathrm{USA}$ ) according to our institutional scanning protocol for breast cancer, consisting of an unenhanced CT of the liver followed by arterial phase at 30 seconds that includes the chest, and portal phase of the abdomen and pelvis at $70 \mathrm{sec}-$ onds. The protocol changed during the study period after the results of another study underwent at our hospital in patients with lung cancer. ${ }^{13}$ The new protocol consisted in an arterial scan at 35 seconds of the liver, followed by a single delayed acquisition at 65 seconds that included chest, abdomen and pelvis. ${ }^{13}$ Finally, 79 scans were performed with the first protocol, and 74 with the new changes.

All patients received a standardized IV injection through a power injector consisting of iomeprol (Iomeron, Bracco) with an iodine concentration of either 350 or $400 \mathrm{mg} \mathrm{I} / \mathrm{mL}$, at a dose of $0.5 \mathrm{~g} \mathrm{I} / \mathrm{kg}$ with a fixed 40 -second duration of injection, followed by a $30-\mathrm{mL}$ saline chaser at the same rate as for the contrast medium.

\section{Image analysis}

Two radiologists (10 and 20 years experienced) independently reviewed CT images at a PACS workstation (Centricity PACS Universal Viewer 6.0, GE Healthcare). Window settings could be changed as desired. Four reading rounds were performed by each reader that are summarized in Figure 1. Firstly, all phases were assessed independently, and unenhanced, arterial and portal phases were sorted from best to worst according to the subjec- 


\section{Evaluation of each phase in a single examination}

153 complete examinations

\begin{tabular}{|l|}
\hline Sort phases from best to worst \\
\hline \hline Score 1,2,3=for scoring phases from \\
best to worst, respectively \\
Score $4=$ at least one lesion was \\
missed at that phase \\
\hline
\end{tabular}

86 examinations showing $\leq 5$ lesions

Sensitivity evaluation

Number of lesions detected at each phase in those studies showing 5 or less

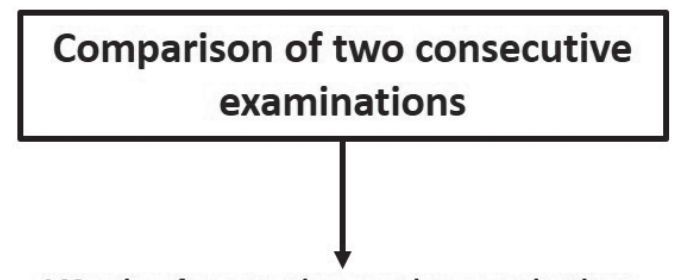

149 pairs of consecutive complete examinations

Score 1=best phase/s for comparison

Score 2=that/those phase/s was/were worse than that/those scored as 1

Score $\mathbf{3}=$ at least one lesion not seen or confidently measured or compared at that phase

FIGURE 1. Flowchart of the reading protocol. There were four steps: sort of phases from best to worst, sensitivity study based only on the reading of examinations having $\leq 5$ metastases, measurement of one target lesion in each examination and evaluation of phases for comparing metastases in 2 consecutive follow-up scans.

tive perception of visualization, conspicuity and delineation of hepatic metastases. If two phases were considered to show lesions in a similar manner, they were scored equally ( 1 if they were better than the other or 2 if worst). If any lesion was missed by one phase, the score was 4 . At this reading, lesions were counted taking into consideration all phases. Lesions were numbered if 5 or less were depicted, and noted if they were more than 5. In a second round, for sensitivity evaluation, readers counted the number of lesions detected in each phase of the 86 examinations that showed 5 lesions or less in the previous reading, randomly presented in a set of 258 complete phases of the liver. The third evaluation round consisted of the comparison of the different phases for assessment of response of hepatic metastases in two paired consecutive scans of the same patient, when available. Both scans were reviewed together, and each phase was scored as 1 if it was the best for compari- son or 2 if response assessment was judged worse compared to other phase. If two phases performed similarly, they were scored equally and, if any lesion was missed or was impossible to confidently be measured for comparison in any phase, it was scored as 3, and considered to alter the assessment of response. Finally, the fourth reading session was the measurement of a target lesion in each examination. For that purpose, a third radiologist, based on previous radiologic reports and her own evaluation, selected and marked a target lesion from each examination in all phases. The lesion she chose was clearly differentiated from others and, if possible, accurately defined in all phases. Readers measured the maximum diameter of the target lesions in the 3 phases of all examinations.

The number of lesions was calculated taking as the reference standard all phases together as well as the follow-up, as is the clinical standard when treating patients with metastatic breast cancer. 


\section{Statistical analysis}

Differences in rating among phases were compared by chi-square test. Weighted kappa statistics was used to measure the degree of agreement between observers for rating different phases. Paired $t$ test was used to compare measurements in each phase. Statistical analysis was performed with the software package IBM SPSS Statistics (version 21, IBM). Statistical significance was set at 0.05 .

\section{Results}

\section{Scoring of individual examinations}

The score of each phase for individual assessment of each examination is presented at Table 1. Portal phase was considered a better phase (score 1) in 68 and $67.3 \%$ by readers 1 and 2 respectively, score 1 for unenhanced phase was 69.3 and $69.9 \%$, while arterial phase was better in 26.1 and $27.5 \%$, with differences being statistically significant $(\mathrm{p}<0.005)$. The percentage of examinations with at least one missed lesion (score 4 ) was 1.3 and $2 \%$ for readers 1 and 2 respectively in unenhanced phase, $6.5 \%$ for both readers in portal phase and 10.5 and $11.8 \%$ for arterial phase (Figure 2,3). No lesion was missed in unenhanced and portal phases together. Agreement between observers for this reading was "good" with kappa values of $0.703,0.726$ and 0.793 for unenhanced, arterial and portal phases, respectively.

\section{Sensitivity evaluation}

During the first reading session in which all phases were considered, there were 5 metastatic lesions or less in 86 examinations $(56.2 \%)$, showing 228 lesions. The sensitivities for readers $1 / 2$ were $97.4 / 96 \%$ for unenhanced, $88.6 / 89 \%$ for arterial and $97.8 / 97.4 \%$ for portal phases, respectively.

\section{Scoring of assessment of response}

Results of comparison of lesions between two consecutive scans are shown in Table 2. Comparison was considered better in $81.2 / 79.2 \%$ of unenhanced, $30.9 / 31.5 \%$ arterial and $71.1 / 69.8 \%$ of portal phases, by readers $1 / 2$, respectively. Agreement between observers for scoring comparisons was "excellent" with values of $0.917,0.964$ and 0.882 for unenhanced, arterial and portal phases, respectively. Considering all 298 readings, in 154 of them $(51.7 \%)$, unenhanced and portal phases were together the best phases (score 1), unenhanced phase
TABLE 1. Distribution of scores of each phase by both readers in 153 examinations

\begin{tabular}{|c|c|c|c|c|c|c|c|}
\hline \multicolumn{8}{|c|}{ Reader 1} \\
\hline & & & & & UNE & CEL & \\
\hline & & & & 1 & 2 & 3 & 4 \\
\hline \multirow{14}{*}{ PORTAL } & \multirow{4}{*}{1} & \multirow{4}{*}{ ARTERIAL } & 1 & 32 & 2 & 2 & 0 \\
\hline & & & 2 & 16 & 20 & 10 & 1 \\
\hline & & & 3 & 9 & 10 & 0 & 0 \\
\hline & & & 4 & 4 & 0 & 0 & 0 \\
\hline & \multirow{4}{*}{2} & \multirow{4}{*}{ ARTERIAL } & 1 & 1 & 0 & 2 & 0 \\
\hline & & & 2 & 6 & 0 & 0 & 0 \\
\hline & & & 3 & 15 & 0 & 0 & 1 \\
\hline & & & 4 & 3 & 0 & 0 & 0 \\
\hline & \multirow{4}{*}{3} & \multirow{4}{*}{ ARTERIAL } & 1 & 0 & 1 & 0 & 0 \\
\hline & & & 2 & 4 & 0 & 0 & 0 \\
\hline & & & 3 & 4 & 0 & 0 & 0 \\
\hline & & & 4 & 0 & 0 & 0 & 0 \\
\hline & \multirow{2}{*}{4} & \multirow{2}{*}{ ARTERIAL } & 2 & 1 & 0 & 0 & 0 \\
\hline & & & 4 & 9 & 0 & 0 & 0 \\
\hline \multicolumn{8}{|c|}{ Reader 2} \\
\hline & & & & \multicolumn{4}{|c|}{ UNENHANCED } \\
\hline & & & & 1 & 2 & 3 & 4 \\
\hline \multirow{13}{*}{ PORTAL } & \multirow{5}{*}{1} & \multirow{4}{*}{ ARTERIAL } & 1 & 30 & 5 & 1 & 0 \\
\hline & & & 2 & 18 & 20 & 7 & 1 \\
\hline & & & 3 & 7 & 12 & 0 & 0 \\
\hline & & & 4 & 6 & 0 & 0 & 0 \\
\hline & & \multirow{4}{*}{ ARTERIAL } & 1 & 2 & 0 & 2 & 1 \\
\hline & \multirow{3}{*}{2} & & 2 & 6 & 0 & 0 & 1 \\
\hline & & & 3 & 14 & 0 & 0 & 0 \\
\hline & & & 4 & 3 & 0 & 0 & 0 \\
\hline & \multirow{3}{*}{3} & \multirow{3}{*}{ ARTERIAL } & 1 & 1 & 0 & 0 & 0 \\
\hline & & & 2 & 3 & 0 & 0 & 0 \\
\hline & & & 3 & 3 & 0 & 0 & 0 \\
\hline & \multirow{2}{*}{4} & \multirow{2}{*}{ ARTERIAL } & 2 & 1 & 0 & 0 & 0 \\
\hline & & & 4 & 9 & 0 & 0 & 0 \\
\hline
\end{tabular}

Scores were 1: best phase; 2 : second best; 3 : worst; 4: at least one lesion was missed at that phase. Figures are number of examinations.

was considered the only best phase in 85 (28.5\%) readings, portal phase in $44(14.8 \%)$, and arterial phase was considered the only best phase for comparison in 3 readings (1\%). In 14 examinations, unenhanced phase allowed to compare lesions that could not be evaluated by portal phase (Figure 2). On a per patient basis, this occurred in at least one scan in 6 out of 36 patients along the study. 

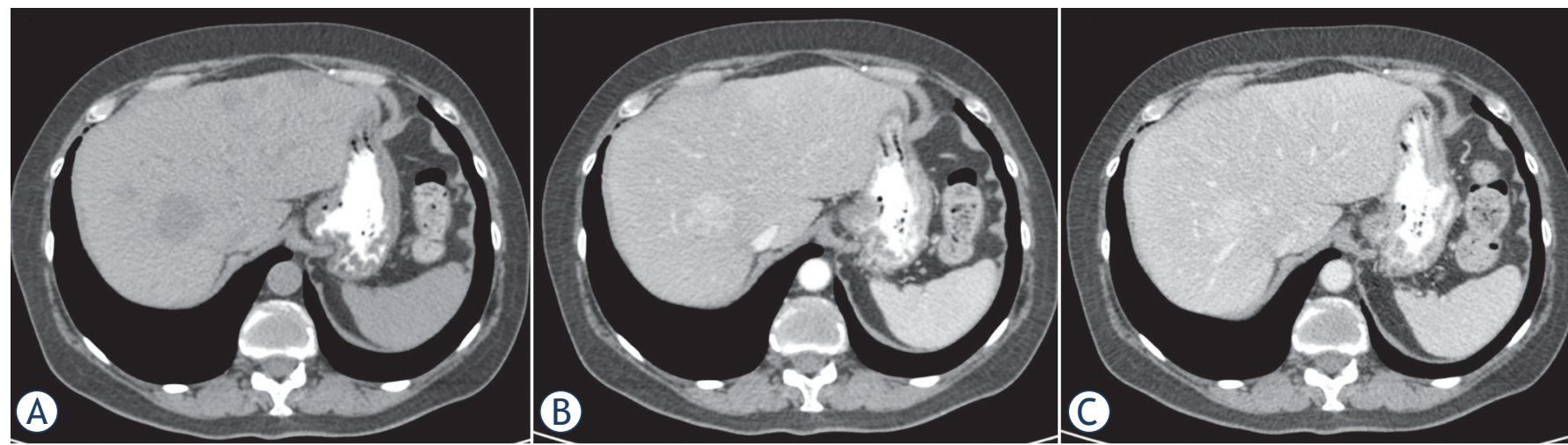
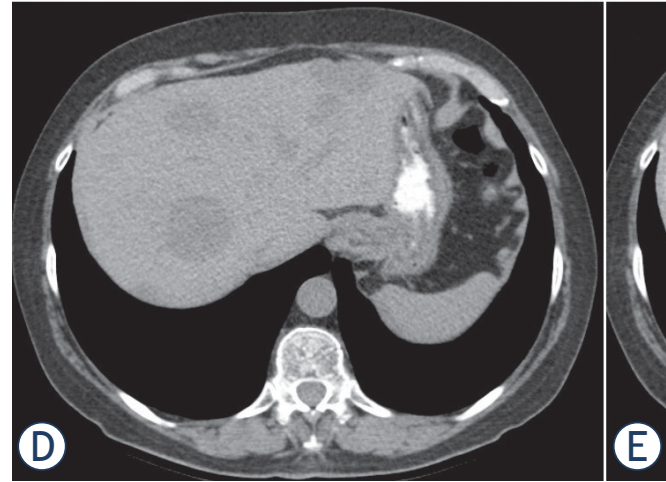

TABLE 2. Distribution of scores of each phase for comparing metastatic lesions in two consecutive scans by both readers in 149 examinations

\begin{tabular}{|c|c|c|c|c|c|c|}
\hline \multicolumn{7}{|c|}{ Reader 1} \\
\hline & & & & \multicolumn{3}{|c|}{ UNENHANCED } \\
\hline & & & & 1 & 2 & 3 \\
\hline \multirow{8}{*}{ PORTAL } & \multirow{3}{*}{1} & \multirow{3}{*}{ ARTERIAL } & 1 & 35 & 6 & 1 \\
\hline & & & 2 & 42 & 20 & 0 \\
\hline & & & 3 & 2 & 0 & 0 \\
\hline & \multirow{3}{*}{2} & \multirow{3}{*}{ ARTERIAL } & 1 & 3 & 0 & 1 \\
\hline & & & 2 & 23 & 0 & 0 \\
\hline & & & 3 & 2 & 0 & 0 \\
\hline & \multirow{2}{*}{3} & \multirow{2}{*}{ ARTERIAL } & 2 & 1 & 0 & 0 \\
\hline & & & 3 & 13 & 0 & 0 \\
\hline
\end{tabular}

\begin{tabular}{|c|c|c|c|c|c|c|}
\hline \multicolumn{7}{|c|}{ Reader 2} \\
\hline & & & & \multicolumn{3}{|c|}{ UNENHANCED } \\
\hline & & & & 1 & 2 & 3 \\
\hline \multirow{8}{*}{ PORTAL } & \multirow{3}{*}{1} & \multirow{3}{*}{ ARTERIAL } & 1 & 39 & 4 & 1 \\
\hline & & & 2 & 34 & 24 & 0 \\
\hline & & & 3 & 2 & 0 & 0 \\
\hline & \multirow{3}{*}{2} & \multirow{3}{*}{ ARTERIAL } & 1 & 1 & 0 & 2 \\
\hline & & & 2 & 26 & 0 & 0 \\
\hline & & & 3 & 2 & 0 & 0 \\
\hline & \multirow{2}{*}{3} & \multirow{2}{*}{ ARTERIAL } & 2 & 1 & 0 & 0 \\
\hline & & & 3 & 13 & 0 & 0 \\
\hline
\end{tabular}

Scores were 1: best phase/s for comparison; 2: that phase was worse for comparing lesions than that scored as 1; 3: at least one lesion was not seen or could not be confidently measured or compared at that phase. Figures are number of examinations.
FIGURE 2. 48-year-old woman with hepatic metastases from breast cancer. Upper row corresponds to previous study and lower row is follow-up. Previous unenhanced phase (A) show more lesions than either the arterial (B) and portal $(C)$ phases. At follow-up 6 weeks later, worsening seen in unenhanced phase (D) cannot be confidently confirmed only by arterial (E) and portal (F) phases.

\section{Size of target lesions}

Maximum diameter of target lesions was statistically significant larger in unenhanced phase than in either arterial or portal phases $(\mathrm{p}<0.001)$ for both observers, as shown in Table 3 (Figure 3). Differences of measurements in unenhanced phase were $\geq 20 \%$ compared to portal phase in $29.7 \%$ of measurements by reader 1 , and in $30.1 \%$ by reader 2 .

\section{Discussion}

Hepatic metastasis from breast cancer appear in more than $50 \%$ of patients with advanced disease, for that reason an adequate detection of the lesions is desirable.

Oncologic guidelines about imaging evaluation of patients with breast cancer lack recommendations about the scanning protocol ${ }^{14}$, while specific radiological recommendations consider only the need of a portal phase. ${ }^{15,16}$ However, some authors 

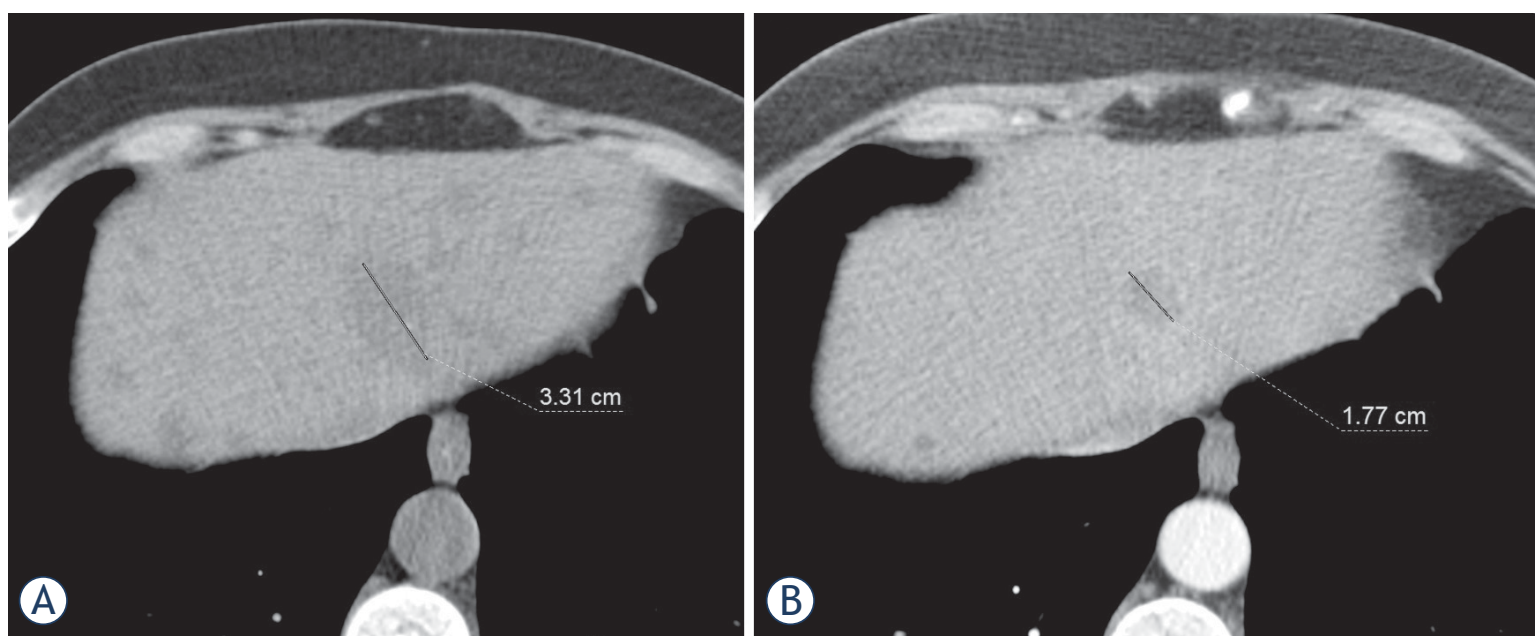

FIGURE 3. 56-year-old woman with hepatic metastases from breast cancer. Measurement of target lesion in unenhanced phase (A) is $33 \mathrm{~mm}$ and in the portal phase (B) is $18 \mathrm{~mm}$, that represent a $45 \%$ difference. A part of the metastatic lesion posteriorly is scarcely seen as a subtle increase attenuation in the portal phase, but both readers failed to consider that area as part of the lesion, and measured only the hypoattenuating component. Note how unenhanced phase also shows other small lesions not seen in the portal phase.

recommend unenhanced phase for these patients ${ }^{17}$ and, in a survey ${ }^{9}$, unenhanced CT was used in $21 \%$ of institutions for breast cancer evaluation. These variations make necessary clarification of this issue.

The primary role of imaging in patients with hepatic metastasis is to get the best delineation of the lesions for their detection and adequate comparison in follow-up. The goal of contrast enhanced $\mathrm{CT}$ of the liver is to get the optimal lesion-to-liver contrast, and for that purpose, different phases after contrast administration may be useful. In our experience, hepatic metastases from breast cancer are sometimes very difficult to differentiate from normal liver in portal phase, while unenhanced images depict them surprisingly well. This fact had already been studied and discussed in several studies carried out in the 1990s.3,7,8,10,11 More recently, a Critically Appraised Topic Review by Sadigh et al. ${ }^{12}$, concluded that unenhanced CT adds a small incremental value to contrast enhanced $\mathrm{CT}$ for the detection of hypervascular metastases, however they remarked that those studies were performed with older CT scanners and contrast infusion technologies which may limit the interpretation of data. Moreover, radiologist's confidence level for detecting lesions hasn't been evaluated in most studies, and it was not clear in most of them whether the CT scan was used as initial staging or for follow-up after treatment. It is also important to consider that the therapeutic arsenal available has expanded and could change the way lesions are seen.
In this setting, our purpose was to evaluate if, with modern equipment and chemotherapy regimens in patients with hepatic metastases from breast cancer, unenhanced CT played any role for detection of lesions, aided in the comparison of studies for response evaluation and if there were significant differences in the size of the lesions.

When readers were asked which phases better showed the lesions, both agreed that portal and unenhanced phases were better than arterial phase in most patients. This is in agreement with older studies evaluating lesion conspicuity in unenhanced phase compared with contrast enhanced ${ }^{7}$, and with arterial and portal phases. ${ }^{3}$ It is important to note that sensitivity of the unenhanced phase was very similar to that of portal phase both in the joined evaluation and in the number of lesions detected, and that unenhanced and portal phases together didn't miss any lesion detected by arterial phase. That has practical implications, since arterial phase could be eliminated in our series maintaining a

TABLE 3. Measurements of target lesions by phase

\begin{tabular}{llll}
\hline & Unenhanced & Arterial & Portal \\
\hline Reader 1 & $27.6 \pm 18.7$ & $23.9 \pm 18.9$ & $24.2 \pm 18.2$ \\
Reader 2 & $27.4 \pm 18.6$ & $24.0 \pm 18.7$ & $24.1 \pm 18.0$
\end{tabular}

Figures are mean \pm standard deviation, in millimeters. Differences between unenhanced and arterial, and unenhanced and portal phases $p<0.0001$ for both readers; differences between arterial and portal phases $p=0.510$ for reader 1 and $p=0.620$ for reader 2 . 
perfect detection rate. Except for one study ${ }^{10}$, that shows a significant greater sensitivity of portal compared to unenhanced phase, the rest of studies evaluating sensitivity for detection of hepatic metastases from breast cancer find similar sensitivities for unenhanced and portal phases, with slight differences favouring one phase or another ${ }^{3,7,8,11}$, as in our case. All these studies, and others including metastases from breast cancer as a proportion of patients included ${ }^{18}$, agree that arterial phase adds less than does unenhanced phase.

In contrast with all the other studies previously referred, this is the first one examining the role of unenhanced phase for evaluating response in follow-up studies of patients with breast cancer, that is one of the most frequent uses of $\mathrm{CT}$ in this population. Since most patients have multiple lesions, detection of any lesion is enough to make a correct diagnosis at the patient level, however, when evaluating response, all the lesions should be detected, and an optimal delineation of them in both examinations being compared is desirable. At this point, the role of unenhanced CT gains relevance, since it was the only phase which allowed to compare all the lesions in $8.7 \%$ of the readings, and was the single best phase in $28.5 \%$. Again, unenhanced and portal phase were both the best phases for comparison in more than $50 \%$, while the arterial phase played a marginal role for this purpose. According to our results, addition of only unenhanced phase of the liver, and not an arterial phase, to a single acquisition portal phase of the chest, abdomen and pelvis is the optimal protocol for better evaluating metastatic disease of the liver in follow-up comparisons in patients with breast cancer. We think that recommendations suggesting the elimination of all phases except for the portal phase $e^{15,16}$ do not have in consideration this important role of unenhanced phase in follow-up, and only consider its marginal role in the overall detection of lesions.

One study ${ }^{3}$ reported that unenhanced CT provided the maximal tumor volume, and our results agreed, obtaining approximately $15 \%$ larger diameter over portal phase. This contrasts with the results of one study ${ }^{19}$ in the evaluation of unenhanced $\mathrm{CT}$ in patients with gastric and colon cancer, in which lesions are shown to be significantly smaller and with much lower sensitivity than in portal phase. Conversely, for the measurement of hepatic metastases of neuroendocrine tumour, unenhanced phase has been suggested as the most reliable. ${ }^{20}$ It must be taken in mind, that when evaluating response by RECIST 1.1, the longest diameter of the target lesions in the phase that it is better shown and more confidently measurable must be used $^{21}$, and in our study it was unenhanced phase in many cases. Moreover, differences in measurements between portal and unenhanced phases are clinically significant since roughly a $30 \%$ of patients showed differences of $\geq 20 \%$.

Our study has several limitations. First, we did not have a pathological confirmation of most lesions as occurs in the usual clinical practice, however, clinical diagnosis and follow-up provided unequivocal behaviour as metastases in all cases. Second, for sensitivity evaluation we lacked a pathological or other imaging technique reference standard, instead we took the evaluation of all phases together as the reference. Although this is a major weakness of our investigation, this approach is nearer to the radiologist's daily work and the real clinical scenario, where radiologists can evaluate all phases together, and some subtle lesions can only be considered after confirming their presence in other phases or in follow-up. Although substantial bias could be derived from this approach, the degree of agreement shown by kappa values supported the reproducibility of our results. It could be argued that some lesions might be missed by all phases, however, their clinical relevance is unknown. Third, a formal sensitivity evaluation was performed only in examinations with 5 lesions or less. The reason for this was that many of the remaining studies had uncountable lesions, and counting all the metastases could be an arduous task, not necessarily representing the real number of detectable lesions, due to confluent metastases and different appearance depending of the phases. However, the proportion of lesions examined by choosing this cut-off of 5 lesions, might give us an approach to the real sensitivity of each phase. Fourth, regarding the measurement differences, whether the greater size in unenhanced images corresponds to tumour infiltration or to other parenchymal changes is not clear. In many cases, with lesions clearly larger in unenhanced phase, margins of the lesions in the other phases were difficult to ascertain and some lesions were actually not visible as shown in the figures. As Zimmerman et al. ${ }^{3}$, we lack a pathologic correlation, except for only one recent case not included in the study, with pathological measurements of resected metastases being much closer to those obtained at unenhanced than at portal phase. Finally, although we have evaluated an adequate number of examinations, the number of patients is limited and they were being treated with a variety of chemotherapy schemes, limiting to take more general conclu- 
sions about the possible role of different therapeutic regimens on the visualization of lesions in one phase or another. In conclusion, our results showed that unenhanced and portal phases of the liver permitted better detection and delineation of metastatic lesions from breast carcinoma, and that unenhanced phase provided the largest diameter and in most cases was the best phase for comparing consecutive CT scans to assess response. For these reasons, our recommendation for institutions that don't do so, is that they use unenhanced CT in addition to the portal phase of the liver for evaluation for patients with breast carcinoma and suspected or known hepatic metastatic disease.

\section{Acknowledgement}

This investigation has been supported by Instituto de Investigación Sanitaria y Biomédica de Alicante (ISABIAL)- (grant no. 19-0259).

\section{References}

1. Bray F, Ferlay J, Soerjomataram I, Siegel RL, Torre LA, Jemal A. Global cancer statistics 2018: GLOBOCAN estimates of incidence and mortality worldwide for 36 cancers in 185 countries. CA Cancer J Clin 2018; 68: 394-424. doi: 10.3322/caac.21492

2. Chikarmane SA, Tirumani SH, Howard SA, Jagannathan JP, DiPiro P. Metastatic patterns of breast cancer subtypes: what radiologists should know in the era of personalized cancer medicine. Clin Radiol 2015; 70: 1-10. doi: 10.1016/j.crad.2014.08.015

3. Zimmerman P, Lu DS, Yang LY, Chen S, Sayre J, Kadell B. Hepatic metastases from breast carcinoma: comparison of noncontrast, arterial-dominant, and portal-dominant phase spiral CT. J Comput Assist Tomogr 2000; 24: 197-203. doi: 10.1097/00004728-200003000-00003

4. Sica GT, Ji H, Ros PR. CT and MR imaging of hepatic metastases. AJR Am J Roentgenol 2000; 174: 691-8. doi: 10.2214/ajr.174.3.1740691

5. Francis IR, Cohan RH, McNulty NJ, Platt JF, Korobkin M, Gebremariam A, et al. Multidetector CT of the liver and hepatic neoplasms: effect of multiphasic imaging on tumor conspicuity and vascular enhancement. AJR Am J Roentgenol 2003; 180: 1217-24. doi: 10.2214/ajr.180.5.1801217

6. Soyer P, Poccard M, Boudiaf M, Abitbol M, HamziL, Panis $\mathrm{Y}$, et al. Detection of hypovascular hepatic metastases at triple-phase helical CT: sensitivity of phases and comparison with surgical and histopathologic findings. Radiology 2004; 231: 413-20. doi: 10.1148/radiol.2312021639

7. DuBrow RA, David CL, Libshitz HI, Lorigan JG. Detection of hepatic metastases in breast cancer: the role of nonenhanced and enhanced CT scanning. J Comput Assist Tomogr 1990; 14: 366-9. doi: 10.1097/00004728199005000-00008

8. Patten RM, Byun JY, Freeny PC. CT of hypervascular hepatic tumors: are unenhanced scans necessary for diagnosis? AJR Am J Roentgenol 1993; 161: 979-84. doi: 10.2214/ajr.161.5.8273641

9. O'Malley ME, Halpern E, Mueller PR, Gazelle GS. Helical CT protocols for the abdomen and pelvis: a survey. AJR Am J Roentgenol 2000; 175: 109-13. doi: 10.2214/ajr.175.1.1750109

10. Frederick MG, Paulson EK, Nelson RC. Helical CT for detecting focal liver lesions in patients with breast carcinoma: comparison of noncontrast phase, hepatic arterial phase, and portal venous phase. J Comput Assist Tomogr 1997; 21: 229-35. doi: 10.1097/00004728-199703000-00012
11. Sheafor DH, Frederick MG, Paulson EK, Keogan MT, DeLong DM, Nelson RC. Comparison of unenhanced, hepatic arterial-dominant, and portal venous-dominant phase helical CT for the detection of liver metastases in women with breast carcinoma. AJR Am J Roentgenol 1999; 172: 961-8. doi: 10.2214/ajr.172.4.10587129

12. Sadigh G, Applegate KE, Baumgarten DA. Comparative accuracy of intravenous contrast-enhanced $\mathrm{CT}$ versus noncontrast $\mathrm{CT}$ plus intravenous contrast-enhanced $\mathrm{CT}$ in the detection and characterization of patients with hypervascular liver metastases: a critically appraised topic. Acad Radiol 2014; 21: 113-25. doi: 10.1016/j.acra.2013.08.023

13. García-Garrigós E, Arenas-Jiménez JJ, Sánchez-Payá J. Best Protocol for combined contrast-enhanced thoracic and abdominal CT for lung cancer: a single-institution randomized crossover clinical trial. AJR Am J Roentgenol 2018; 210: 1226-34. doi: 10.2214/ajr.17.19185

14. Cardoso F, Senkus E, Costa A, Papadopoulos E, Aapro M, Andre F, et al. 4th ESO-ESMO International Consensus Guidelines for Advanced Breast Cancer (ABC 4). Ann Oncol 2018; 29: 1634-57. doi: 10.1093/annonc/mdy192

15. Barter S, Britton P. Breast cancer. In: Nicholson T, editor. Recommendations for cross-sectional imaging in cancer management. Second edition. London: The Royal College of Radiologists; 2014.

16. Johnson PT, Bello JA, Chatfield MB, Flug JA, Pandharipande PV, Rohatgi S, et al. New ACR choosing wisely recommendations: judicious use of multiphase abdominal CT protocols. J Am Coll Radiol 2019; 16: 56-60. doi: 10.1016/j. jacr.2018.07.026

17. Siewert B, Sosna J, McNamara A, Raptopoulos V, Kruskal JB. Missed lesions at abdominal oncologic CT: lessons learned from quality assurance. Radiographics 2008; 28: 623-38. doi: 10.1148/rg.283075188

18. Oliver JH 3rd, Baron RL, Federle MP, Jones BC, Sheng R. Hypervascular liver metastases: do unenhanced and hepatic arterial phase $C T$ images affect tumor detection? Radiology 1997; 205: 709-15. doi: 10.1148/radiology.205.3.9393525

19. Jee HB, Park MJ, Lee HS, Park MS, Kim MJ, Chung YE. Is non-contrast CT adequate for the evaluation of hepatic metastasis in patients who cannot receive iodinated contrast media? PLoS One 2015; 10: e0134133. doi: 10.1371/journal.pone.0134133

20. Huh J, Park J, Kim KW, Kim HJ, Lee JS, Lee JH, et al. Optimal phase of dynamic computed tomography for reliable size measurement of metastatic neuroendocrine tumors of the liver: comparison between pre- and post-contrast phases. Korean J Radiol 2018; 19: 1066-76. doi: 10.3348/ kjr.2018.19.6.1066

21. Eisenhauer EA, Therasse P, Bogaerts J, Schwartz LH, Sargent D, Ford R, et al. New response evaluation criteria in solid tumours: revised RECIST guideline (version 1.1). Eur J Cancer 2009; 45: 228-47. doi: 10.1016/j.ejca.2008.10.026 